\title{
Electromagnetic cavity with arbitrary $Q$ and small modal volume without a complete photonic bandgap
}

\author{
M. R. Watts, S. G. Johnson, H. A. Haus, and J. D. Joannopoulos \\ Research Laboratory of Electronics and Center for Materials Science and Engineering, Massachusetts Institute of Technology, \\ Cambridge, Massachusetts 02139
}

Received June 25, 2002

\begin{abstract}
We show how an electromagnetic cavity with arbitrarily high $Q$ and small (bounded) modal volume can be formed in two or three dimensions with a proper choice of dielectric constants. Unlike in previous work, neither a complete photonic bandgap nor a trade-off in mode localization for $Q$ is required. Rather, scattering and radiation are prohibited by a perfect mode match of the TE-polarized modes in each subsection of a Bragg resonator. $Q$ values in excess of $10^{5}$ are demonstrated through finite-difference time-domain simulations of two- and three-dimensional structures with modal areas or volumes of the order of the wavelength squared or cubed. () 2002 Optical Society of America
\end{abstract}

OCIS codes: $050.2230,230.5750$.

Electromagnetic resonant cavities, which trap light within a finite volume for many optical periods, are an essential component of many important optical devices and effects, from lasers to filters to nonlinear switches. Cavities are characterized by two quantities: the modal volume, $V, 1,2$ and the quality factor, $Q$ [the number of optical periods to decay by $e^{-2 \pi}$ (Refs. 3 and 4)]. In many applications (e.g., lasers, filters, and nonlinear devices), high $Q$ and small $V$ are desirable. We describe an analytical mode-matching mechanism in two and three dimensions that, in principle, allows arbitrarily high $Q$ to be achieved with a bounded modal area or volume of the order of the wavelength squared or cubed. Our mechanism does not employ a complete photonic bandgap, ${ }^{5}$ which, at the expense of complex structures in three dimensions, was the only previously known route to such results. ${ }^{6}$ Unlike with other designs to improve cavity $Q$ in the absence of a complete bandgap, ${ }^{4,7-11}$ we need not increase the modal volume $V$ as a trade-off for arbitrarily high $Q$-indeed, our $V$ is roughly independent of $Q$.

When a waveguide mode encounters an interface, there are normally radiation losses. However, if the guided mode in one section can be expressed purely as a linear combination of the forward and backward guided modes of the other section, there will be reflections without scattering or radiation. A quarter-wave stack and a Fabry-Perot cavity (as in Fig. 2, below) can then be constructed through a series of such junctions to trap a mode that decays exponentially in all directions. The operating characteristics of the cavity are then completely described by an equivalent onedimensional system. In this Letter we demonstrate how to achieve such mode matching with a simple analytic condition on the dielectric constants and present examples of such high- $Q$ cavities in two and three dimensions. (A similar mode-matching proof, in two dimensions only and without application to cavities, was given in Ref. 12 to eliminate losses from index-confined Bragg mirrors.) To demonstrate this result, we begin by using the wave equation to develop necessary conditions on both the materials and the modes for the junction of two step-index waveguides (depicted in Fig. 1) to be radiation free. We then show that a superposition of forward- and backward-propagating guided modes entirely satisfies the boundary conditions, precluding any coupling to radiation modes.

For guided modes propagating along $\hat{z}$, the electric field $\mathbf{E}$ in a waveguide has $z$ dependence $\exp (-j \beta z)$ and obeys the wave equation

$$
\left(\nabla_{T}^{2}+\mu_{0} \epsilon_{i} \omega^{2}-\beta^{2}\right) \mathbf{E}=0
$$

in the piecewise-uniform regions $i$, where $\nabla_{T}^{2} \equiv \nabla^{2}-\partial_{z}^{2}$ denotes the transverse Laplacian (similarly for $\tilde{\mathbf{E}}, \tilde{\beta}$, and $\tilde{\epsilon}_{i}$ and in the second waveguide). Since the magnetic and transverse electric fields must be continuous across the junction, the transverse-mode profiles $\mathbf{E}_{T}$ and $\tilde{\mathbf{E}}_{T}$ must be at least componentwise proportional if the field solutions are to be composed solely of guided modes, so we require that Eq. (1) be the same for both waveguides. Thus, $\epsilon_{i} \omega^{2}-\beta^{2} / \mu_{0}=\tilde{\epsilon}_{i} \omega^{2}-\tilde{\beta}^{2} / \mu_{0}$, which implies that

$$
\epsilon_{1}-\epsilon_{2}=\tilde{\epsilon}_{1}-\tilde{\epsilon}_{2}
$$

Equation (2), however, is incompatible with the condition of continuity on $\hat{n} \cdot\left(\epsilon_{i} \mathbf{E}_{i}\right)$, except in the trivial case of $\epsilon_{i}=\tilde{\epsilon}_{i}$ or when the normal component of the

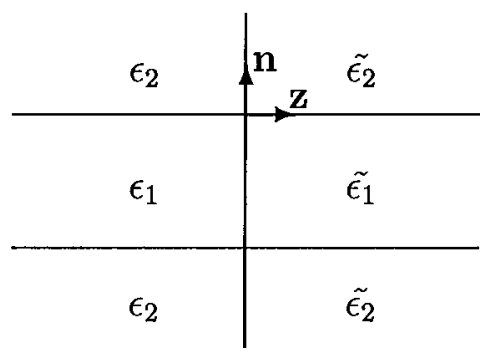

Fig. 1. Schematic junction of two step-index waveguides. 
$\mathbf{E}_{i}$ is zero. Therefore, we immediately restrict our attention to the case in which Eq. (2) is satisfied and the electric field is TE (transverse electric, i.e., purely parallel to the waveguide walls).

Under these conditions, the TE fields in the two waveguides satisfy the same differential equations and have the same boundary conditions and therefore permit identical (or proportional) solutions. It remains to be shown that a superposition of only these guided modes satisfies all the boundary conditions at the junction. For this to hold, we must include the magnetic field, whose transverse components are given by Faraday's law in the TE case:

$$
\hat{z} \frac{\partial}{\partial z} \times \mathbf{E}_{T}=-j \omega \mu_{0} \mathbf{H}_{T}=-j \beta \hat{z} \times \mathbf{E}_{T}
$$

Now, we take as a trial solution a superposition of a forward- and a backward-propagating mode in the left-hand waveguide and a single forward-propagating mode in the right-hand waveguide. At the boundary, it is necessary and sufficient that the transverse field profiles be continuous, and thus

$$
\begin{aligned}
& \mathbf{E}_{T}(1+r)=t \cdot \tilde{\mathbf{E}}_{T}, \\
& \mathbf{H}_{T}(1-r)=t \cdot \tilde{\mathbf{H}}_{T},
\end{aligned}
$$

where $|r|^{2}$ and $|t|^{2}$ are the reflection and transmission coefficients, respectively. By applying Eq. (3) to Eq. (5) and solving for $r$, given from above that the TE field profiles are proportional, we find that all boundary conditions are satisfied with the usual reflection coefficient ${ }^{7}$ :

$$
r=\frac{n_{\mathrm{eff}}-\tilde{n}_{\mathrm{eff}}}{n_{\mathrm{eff}}+\tilde{n}_{\mathrm{eff}}}
$$

for the effective indices $n_{\text {eff }} \equiv \beta C / \omega$. That is, the unique solution of Maxwell's equations consists of forward- and backward-propagating modes of normalized amplitudes 1 and $r$, respectively, in the left-hand guide and a single forward-propagating mode in the right-hand guide of normalized amplitude $t=1+r$.

In summary, we have shown that if Eq. (2) is satisfied and the excited mode is purely TE, all boundary conditions at the junction are necessarily satisfied by guided-mode solutions and the junction is radiation free. In two dimensions, one can always choose the electric field to be TE polarized (polarization everywhere out of the plane in Fig. 1). In three dimensions, for cylindrical waveguides, the azimuthally polarized $\mathrm{TE}_{0 l}$ modes are purely $\mathrm{TE}$ : Their polarization is everywhere directed along $\hat{\phi}$ (parallel to the walls). Because there are only reflections, the system is effectively one dimensional and so a quarter-wave stack (thicknesses $\pi / 2 \beta$ and $\pi / 2 \tilde{\beta}$ ) with a quarter-wave defect ${ }^{13}$ can be used to optimally confine light in the axial direction without sacrificing lateral confinement or $Q$. In fact, the only limitations on the cavity $Q$ will result from the limited number of Bragg layers and the finite extent of the cladding, as well as fabrication imperfections.

The theory presented above is exact, but we also present numerical results to illustrate the theory and the effects of finite system size. We consider the structure, depicted in Fig. 2, comprising a quarter-wave stack of waveguides with a quarter-wave Fabry-Perot defect in the center of the structure, with $N$ Bragg bilayers on either side. The core width $a$ is arbitrary, but here it was chosen to ensure single-mode guidance and satisfies $a / \lambda=0.2839$. The indices were chosen to satisfy Eq. (2), with indices $n_{1}=3, n_{2}=\sqrt{6}, \tilde{n}_{1}=2$, and $\tilde{n_{2}}=1$; the associated effective indices of the guide sections were calculated to be $n_{\text {eff }} \cong 2.8141$ and $\tilde{n}_{\text {eff }} \cong 1.7086$. The field (Fig. 2) and its $Q$ were computed by finite-difference time-domain simulation. ${ }^{14}$ Two variations of the structure were considered: one with $N=10$ bilayers and the other with $N=15$ bilayers. In each case, the cladding width $T$ was varied and the cavity $Q$ was determined from the energy decay in the cavity. The values of $Q$ increase nearly expontially with the cladding width (Fig. 3) until maxima are reached. Comparisons with $Q$ of one-dimensional stacks with

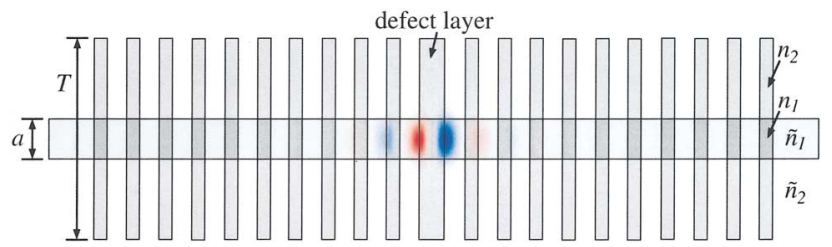

Fig. 2. Two dimensional $(x-z)$ Fabry-Perot cavity, with $\epsilon$ in gray scale, confining a TE mode whose field $E_{y}$ is shown as blue (red) for negative (positive). The cavity consists of alternating index-guided waveguides with core/cladding indices $n_{1} / n_{2}$ and $\tilde{n}_{1} / \tilde{n}_{2}$, where the core has width $a$ and the $n_{2}$ regions have finite width $T$. The indices satisfy Eq. (2), which ensures zero radiation losses at the waveguide interfaces.

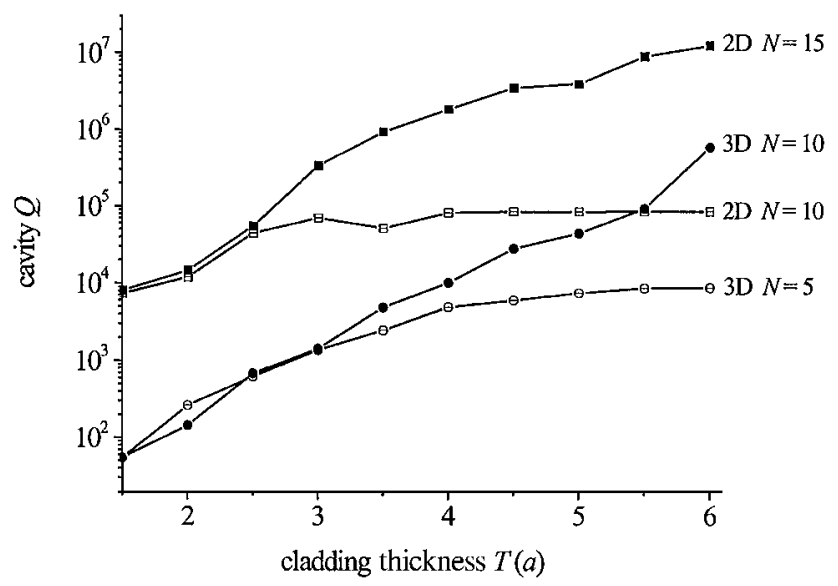

Fig. 3. $Q$ as a function of cladding thickness $T$ for the two-dimensional cavity of Fig. 2 and the three-dimensional cavity for Fig. 4, below, for different numbers $N$ of Bragg periods on either side of the cavity. $Q$ increases exponentially with $T$ or $N$, depending on which one is limiting the $Q$. 


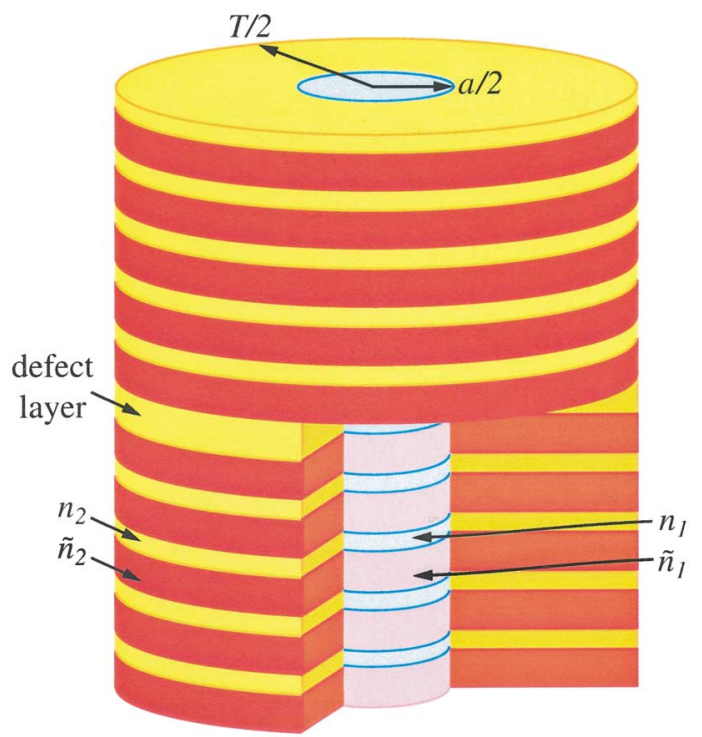

Fig. 4. Schematic of a three-dimensional Fabry-Perot cavity consisting of alternating index-guide cylindrical waveguides stacked in the $z$ direction with core/cladding indices $n_{1} / n_{2}$ and $\tilde{n}_{1} / \tilde{n}_{2}$, where the core (seen in cutaway at the bottom) has diameter $a$ and the cladding has diameter $T$. When the indices satisfy Eq. (2), the $\mathrm{TE}_{01}$ mode does not radiate at the waveguide interfaces.

the same effective indices reveal that maximum $Q$ obtained in the simulations are precisely those of the equivalent one-dimensional structures, thereby demonstrating that $Q$ are limited only by $N$ when the lateral extent of the structure is sufficient. In addition to $Q$, the modal area of this structure, $A \sim(0.31 \lambda)^{2}$, was calculated according to the definition $A=\int \epsilon|\mathbf{E}|^{2} \mathrm{~d} A / \max \left(\epsilon|\mathbf{E}|^{2}\right)^{1,2}$

Similar results are possible in three dimensions if one employs, e.g., the $\mathrm{TE}_{01}$ mode of cylindrical structure. A schematic of such a structure is depicted in Fig. 4. Here, again, we consider a quarter-wave stack with a quarter-wavelength defect and the same indices as in our two-dimensional example. In this case we employ a shorter wavelength (or a larger core diameter a) with $a / \lambda=0.5182$ to support a $\mathrm{TE}_{01}$ mode, since this mode is not fundamental. The effective indices of the $\mathrm{TE}_{01}$ mode are then $n_{\text {eff }} \cong 2.518$ and $\tilde{n}_{\text {eff }} \cong 1.158$. We again measure the cavity $Q$ as a function of $N$ and $T$ by finite-difference time-domain simulations, and the results are shown in Fig. 3. On account of the higher effective-index contrast, only $N=10$ is required for $Q$ of greater than $10^{5}$ (with a cladding diameter $T=6 a$ ). The modal volume, $V, 1,2$ of the structure was calculated and found to be $V \sim(0.406 \lambda)^{3}$.

In summary, we have shown that by satisfying a simple criterion on the indices and employing pure TE modes, we can construct resonant cavities with arbitrary $Q$ and small (bounded) modal volume in both two and three dimensions without a complete photonic bandgap. Moreover, we have demonstrated exemplary designs based on a standard quarter-wave stack with a center quarter-wave defect, exhibiting $Q$ of more than $10^{5}$ with modest system sizes. The $Q$ values of such cavities are limited by the system size (e.g., $N$ and $T$ above), the degree to which Eq. (2) on $\epsilon$ can be satisfied, material absorption, surface roughness, and other disorder. More-complicated mirror structures (e.g., non-quarter-wave shifted) and (or) multiple defect sites may be used to tailor the transmission and dispersion characteristics of a cavity. In practice, to relax the material constraints, one may combine our mode-matching technique with some other strategy for increasing $Q$, such as one based on mode delocalization, or perhaps use mode matching in one dimension and two-dimensional photonic bandgap confinement in the others.

This work was supported in part by the Materials Research Science and Engineering Center program of the National Science Foundation under awards DMR-9808941 and DMR-9400334. M. Watts' e-mail address is mwatts@mit.edu.

\section{References}

1. E. M. Purcell, Phys. Rev. B 69, 681 (1946).

2. R. Coccioli, M. Boroditsky, K. W. Kim, Y. Rahmat-Samii, and E. Yablonovitch, IEE Proc. Optoelectron 145, 391 (1998).

3. H. A. Haus, Waves and Fields in Optoelectronics (Prentice-Hall, Englewood Cliffs, N.J., 1984), Chap. 7.

4. P. R. Villeneuve, S. Fan, S. G. Johnson, and J. D. Joannopoulos, IEE Proc. Optoelectron. 145, 384 (1998).

5. J. D. Joannopoulos, R. D. Meade, and J. N. Winn, Photonic Crystals: Molding the Flow of Light (Princeton U. Press, Princeton, N.J., 1995).

6. P. R. Villeneuve, S. Fan, and J. D. Joannopoulos, Phys. Rev. B 54, 7837 (1996).

7. B. E. A. Saleh and M. C. Teich, Fundamentals of Photonics (Wiley, New York, 1991).

8. H. Benisty, D. Labilloy, C. Weisbuch, C. J. M. Smith, T. F. Krauss, D. Cassagne, A. Béraud, and C. Jouanin, Appl. Phys. Lett. 76, 532 (2000).

9. S. G. Johnson, S. Fan, A. Mekis, and J. D. Joannopoulos, Appl. Phys. Lett. 78, 3388 (2001).

10. S. G. Johnson, A. Mekis, S. Fan, and J. D. Joannopoulos, Computing Sci. Eng. 3, 38 (2001).

11. J. Vučković, M. Lončar, H. Mabuchi, and A. Scherer, Phys. Rev. E 65, 016608 (2002).

12. J. Čtyroký, J. Opt. Soc. Am. A 18, 435 (2001).

13. P. Yeh, Optical Waves in Layered Media (Wiley, New York, 1988).

14. K. S. Kunz and R. J. Luebbers, The Finite-Difference Time-Domain Method for Electromagnetics (CRC, Boca Raton, Fla., 1993) 